\title{
Monosynaptic Tracing Success Depends Critically on Helper Virus Concentrations
}

\author{
Thomas K. Lavin, Lei Jin, Nicholas E. Lea and lan R. Wickersham* \\ McGovern Institute for Brain Research, Massachusetts Institute of Technology, Cambridge, MA, United States
}

Monosynaptically-restricted transsynaptic tracing using deletion-mutant rabies virus (RV) has become a widely used technique in neuroscience, allowing identification, imaging, and manipulation of neurons directly presynaptic to a starting neuronal population. Its most common implementation is to use Cre mouse lines in combination with Cre-dependent "helper" adeno-associated viral vectors (AAVs) to supply the required genes to the targeted population before subsequent injection of a first-generation $(\Delta \mathrm{G})$ rabies viral vector. Here we show that the efficiency of transsynaptic spread and the degree of nonspecific labeling in wild-type control animals depend strongly on the concentrations of these helper AAVs. Our results suggest practical guidelines for achieving good results.

Keywords: rabies, virus, monosynaptic tracing, AAV (adeno-associated virus), circuit tracing

\section{INTRODUCTION}

OPEN ACCESS

Edited by:

Dirk Feldmeyer,

Julich Research Centre, Germany

Reviewed by: Francesco Ferraguti, Innsbruck Medical University, Austria Edward M. Callaway, Salk Institute for Biological Studies, United States

${ }^{*}$ Correspondence: lan R. Wickersham wickersham@mit.edu

Received: 20 August 2019 Accepted: 23 January 2020 Published: 14 February 2020

Citation:

Lavin TK, Jin L, Lea NE and Wickersham IR (2020) Monosynaptic Tracing Success Depends Critically on Helper Virus Concentrations. Front. Synaptic Neurosci. 12:6. doi: 10.3389/fnsyn.2020.00006
Rabies virus (RV) has proven useful for neuroscience, because of its natural behavior of spreading between synaptically-connected neurons [although both the mechanism of transsynaptic spread and the true degree of its synaptic specificity remain unclear (Luo et al., 2018; Beier, 2019)] in an apparently exclusively retrograde direction (in the central nervous system, whereas in primary sensory neurons it appears to be bidirectional (Bauer et al., 2014; Zampieri et al., 2014). This has allowed it to serve as a useful tool for mapping synaptic connections, usually in the context of "monosynaptic tracing," which refers to the use of a modified RV to label neurons that are, putatively, directly presynaptic to a targeted population of neurons (Wickersham et al., 2007b). It relies on the use of a RV to which two modifications have been made. First, in order to render it incapable of spread between neurons without assistance, one (or more) of its genes has been deleted; in first-generation vectors, this is the glycoprotein gene, denoted "G" (Wickersham et al., 2007a). Second, in order to allow selective targeting of the initial infection to the group of neurons of interest, the viral particles are coated with the envelope protein of a different virus (the avian-endemic retrovirus "ASLV-A"; the envelope protein of which is called "EnvA"), rendering the virus incapable of infecting mammalian neurons without assistance (Wickersham et al., 2007b). In the targeted neuronal population, two exogenous proteins must be expressed before injection of the RV: the receptor for EnvA [a quail cell surface protein called "TVA" (Bates et al., 1993)], to allow the modified RV to infect the starting cells, and the deleted viral gene(s) [G, in the case of first-generation (" $\Delta G$ ") vectors]. While this can be achieved by single-cell transfection techniques (Marshel et al., 2010; Rancz et al., 2011; Wertz et al., 2015; Rompani et al., 2017), the much more accessible and widely used implementation is to use Cre (Sauer and Henderson, 1988)-dependent adeno-associated viral vectors (“AAVs"; Atasoy et al., 2008; Wall et al., 2010; Watabe-Uchida et al., 2012; Kohara et al., 2014; Beier et al., 2015; Liu et al., 2017; Ährlund-Richter et al., 2019; Szonyi et al., 2019; Zhang et al., 2019) in combination with a Cre mouse line, in order to map inputs to 
a Cre-expressing group of neurons (see Figure 1). This approach has been used in a large number of studies and contributed considerably to our understanding of the organization of many circuits within the mammalian nervous system.

Within this basic paradigm of using Cre-dependent "helper" AAVs to provide the genes required for monosynaptic tracing, many variations are possible. A major consideration in design of such experiments is the mismatch between the minuscule amount of TVA required for successful initial infection of the starting cells, because of the high sensitivity of the EnvA-TVA interaction (Federspiel et al., 1994; Seidler et al., 2008), and the high levels of $\mathrm{G}$ that appear to be required for efficient spread of the virus from the starting cells to the putatively presynaptic cells. This causes a problem: to achieve acceptable levels of transsynaptic spread, a high concentration of AAV may be found to be necessary; however, because it appears that all putatively Cre-dependent AAVs "leak" because of spontaneous recombination of some fraction of virions' genomes, even at the plasmid DNA stage [Kimberly Ritola, personal communication; unpublished results from our laboratory; and see also a very recent article on this issue (Fischer et al., 2019)], a high concentration of an AAV expressing TVA can result in an unacceptably high level of "background" labeling of cells by the RV in wild-type mice. Different groups have taken different approaches to dealing with this, including using two separate
Cre-dependent AAVs so that $G$ is expressed at a presumably higher level than TVA (Watabe-Uchida et al., 2012) and/or using a low-affinity mutant of TVA (Miyamichi et al., 2013; Sakurai et al., 2016).

Our current approach is to use a mixture of two AAVs, described first in Liu et al. (2017), which are coupled with the tetracycline transactivator system (Gossen and Bujard, 1992). The first AAV is Cre-dependent and expresses, in Cre-expressing cells, TVA (transmembrane isoform; Bates et al., 1993; Young et al., 1993; Jha et al., 2011), EGFP (Cormack et al., 1996), and the tetracycline transactivator ("tTA"); the second is not directly Cre-dependent but simply expresses both G (SAD B19 strain; Conzelmann et al., 1990) and the blue fluorophore mTagBFP2 (Subach et al., 2011) under the control of the tetracycline response element. Expression of tTA from the first AAV drives expression of $\mathrm{G}$ from the second AAV in the same cells. Note that this uses the "TET-OFF" system so that no tetracycline (or doxycycline, etc.) needs to be added in order to make the system work.

The use of this combination has several intended advantages. First, the use of a two-AAV combination allows the concentrations of the vectors encoding TVA and G to be independently titrated. Second, the tTA-TRE system should provide amplification of the level of $G$ expression with respect to the level of expression of the genes in the first AAV, so that

\section{Day 0: inject helper AAVs

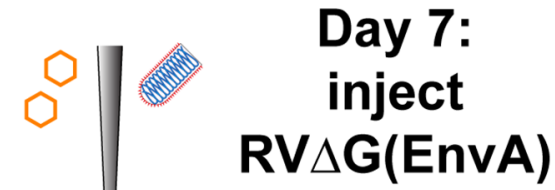 \\ Day 14: \\ examine tissue}

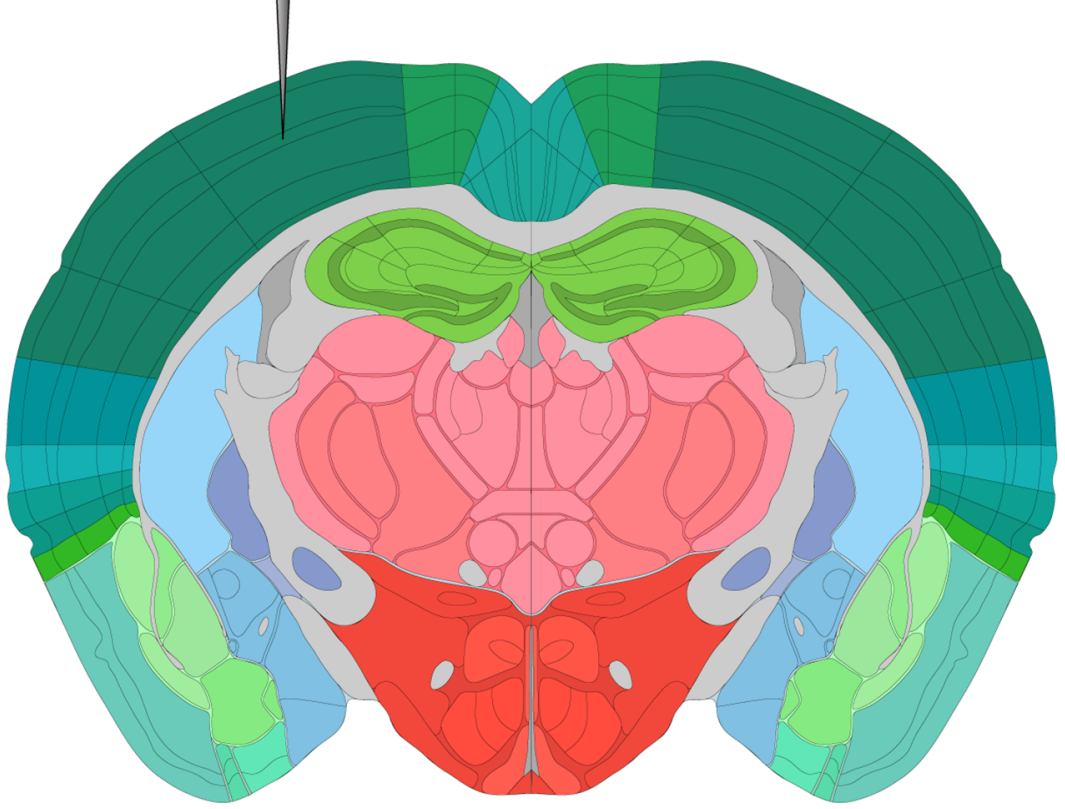

FIGURE 1 | Strategy for monosynaptic tracing with helper adeno-associated viral vectors (AAVs). Helper viruses are injected in a Cre mouse, or a Cre-negative mouse in the case of control experiments, then rabies virus (RV; $\Delta \mathrm{G}$, EnvA-coated, expressing mCherry) is injected in the same location subsequently. While different labs have used various intervals and survival times, we used a 7-day interval between AAV and RV injections, and another 7-day interval between RV injection and perfusion, in all experiments for this article. Brain image adapted with permission from the Allen Mouse Brain Atlas. 
the TVA/EGFP/tTA virus can be titrated down to very low concentration to result in low background labeling in wild-type mice but with the $G$ expression level still high enough to result in plentiful transsynaptic spread of the RV. Third, the use of the tet transactivator system can also allow the expression of $G$ (and mTagBFP2) to be turned off (or potentially titrated) by administration of doxycycline after transsynaptic spread has taken place, in order to mitigate toxicity to the starting cells, although we have not done this in any of the work presented in this article.

We have recently published a detailed step-by-step protocol for monosynaptic tracing using these viruses for monosynaptic tracing with Cre mice (Lavin et al., 2019b). Here we present results of our titration experiments to test the effects of using different dilutions of the helper viruses, to show the reasons for the specific concentrations that we recommend. We found that the two-helper combination described above and in Liu et al. (2017) gives much better results than the simpler single AAV which we described earlier (Kohara et al., 2014), which did not allow independent optimization of transsynaptic tracing efficiency and minimization of background labeling in Cre-negative mice. We also found that excessively high titers of the helper viruses gave very poor results, suggesting that preventing toxicity due to overly high expression of the helper virus genes [or perhaps simply due to either direct toxicity of the AAVs or an immune reaction to them (Hirsch et al., 2011; Bockstael et al., 2012; Flotte and Büning, 2018; Hinderer et al., 2018; Hordeaux et al., 2018a,b; Rabinowitz et al., 2019)] is as minuscule as ensuring sufficient expression of them. Finally, and most practically, we suggest specific concentrations of the helper viruses that gave best results in the Cre line in which we performed the titration and that should serve either as a likely choice of parameters for end-users or as a promising starting point for much more limited titration series to be done as pilot experiments when targeting other cell types in other Cre lines.

\section{MATERIALS AND METHODS}

All experiments involving animals were conducted according to NIH guidelines and approved by the MIT Committee for Animal Care. Mice were housed 1-5 per cage under a normal light/dark cycle for all experiments.

\section{Viruses}

Cloning of AAV genome plasmids pAAV-synP-FLEX-splitTVAEGFP-B19G (Addgene 52473), pAAV-syn-FLEX-splitTVAEGFP-tTA (Addgene 100798), and pAAV-TREtight-mTagBFP2B19G (Addgene 100799) has been described (Kohara et al., 2014; Liu et al., 2017). These genomes were packaged in serotype 1 AAV capsids by, and are available for purchase from, Addgene (catalog numbers 52473-AAV1, 100798-AAV1, and 100799AAV1). The titers of the AAVs, as determined by Addgene by qPCR, were as follows:

- AAV1-synP-FLEX-splitTVA-EGFP-B19G (lot \#v14715): $2.4 \times 10^{13} \mathrm{gc} / \mathrm{ml}$
- AAV1-syn-FLEX-splitTVA-EGFP-tTA (lot \#v15287): $1.7 \times 10^{13} \mathrm{gc} / \mathrm{ml}$

- AAV1-TREtight-mTagBFP2-B19G (lot \#v14716): $3.2 \times 10^{13} \mathrm{gc} / \mathrm{ml}$.

Cloning of pRV $\triangle \mathrm{G}-4 \mathrm{FLPo}$ (Matsuyama et al., 2019; Addgene 122050) and pRV $\Delta$ G-4mCherry (Weible et al., 2010; Addgene 52488) have been described. Production of EnvA-enveloped RV RV $\Delta \mathrm{G}-4 \mathrm{mCherry}($ EnvA) (Kohara et al., 2014) was done as described previously (Wickersham et al., 2010; Wickersham and Sullivan, 2015; Chatterjee et al., 2018) but using helper plasmids pCAG-B19N (Addgene \#59924), pCAG-B19P (Addgene \#59925), pCAG-B19G (Addgene \#59921), pCAG-B19L (Addgene \#59922), and pCAG-T7pol (Addgene \#59926) for the rescue step (Chatterjee et al., 2018). The final titers were $5.82 \times 10^{9}$ infectious units/ml for RV $\Delta \mathrm{G}-4 \mathrm{FLPo}($ EnvA) and $1.70 \times 10^{10}$ infectious units/ml for RV $\Delta \mathrm{G}-4 \mathrm{mCherry}(\mathrm{EnvA})$, as determined by infection of TVA-expressing cells as described previously (Wickersham et al., 2010; Matsuyama et al., 2019).

Helper AAVs were diluted in Dulbecco's phosphate-buffered saline (DPBS; Fisher, 14-190-250) by the desired dilution factors (see main text). In the case of AAV-syn-FLEX-splitTVA-EGFPtTA and AAV-TREtight-mTagBFP2-B19G, the two viruses were combined (after dilution, when applicable) in a 50/50 ratio by volume before injection.

\section{Mouse Strains}

Adult mice of both sexes were used. For compatibility with other projects in the laboratory, the PV-Cre (Jackson 017320) and DAT-Cre (Jackson 006660) used were also heterozygous for the FLP-dependent tdTomato reporter line Ai65F (Daigle et al., 2018); obtained in this case by crossing the Cre- and FLP-dependent tdTomato double-reporter line Ai65D (Madisen et al., 2015; Jackson Laboratory 021875) to the Cre deleter line Meox2-Cre (Tallquist and Soriano, 2000; Jackson Laboratory 003755), then breeding out the Meox2-Cre allele, resulting in a reporter line for which only FLP is required for expression of tdTomato). For those mice in which RV $\Delta$ G-4FLPo was used, the reporter allele was necessary for reporting RV activity; for those in which RV $\Delta \mathrm{G}-4 \mathrm{mCherry}$ was used, the presence of this reporter allele was irrelevant. For Cre-negative control injections using RV $\triangle \mathrm{G}-4 \mathrm{FLPo}$, the Ai65F line was used. For Cre-negative control injections using RV $\Delta \mathrm{G}-4 \mathrm{mCherry}$, either Ai65F or the Cre-dependent reporter line Ail4 (Madisen et al., 2010) was used; in these cases, the presence of the reporter alleles was again irrelevant.

\section{Stereotaxic Injections}

For pilot studies (Figures 2-4), we injected $300 \mathrm{nl}$ of helper AAV solution into primary somatosensory cortex (coordinates with respect to bregma: $\mathrm{AP}=-0.58 \mathrm{~mm}, \mathrm{LM}=3.00 \mathrm{~mm}$, $\mathrm{DV}=-1.00 \mathrm{~mm}$ ) of anesthetized adult mice as described (Lavin et al., 2019b), using a stereotaxic instrument (Stoelting Co., 51925) and custom injection apparatus consisting of a hydraulic manipulator (Narishige, MO-10) with head-stage coupled via custom adaptors to a wire plunger advanced through pulled glass capillaries (Drummond, Wiretrol II) back-filled with mineral oil and front-filled with viral vector solution. Seven days after 

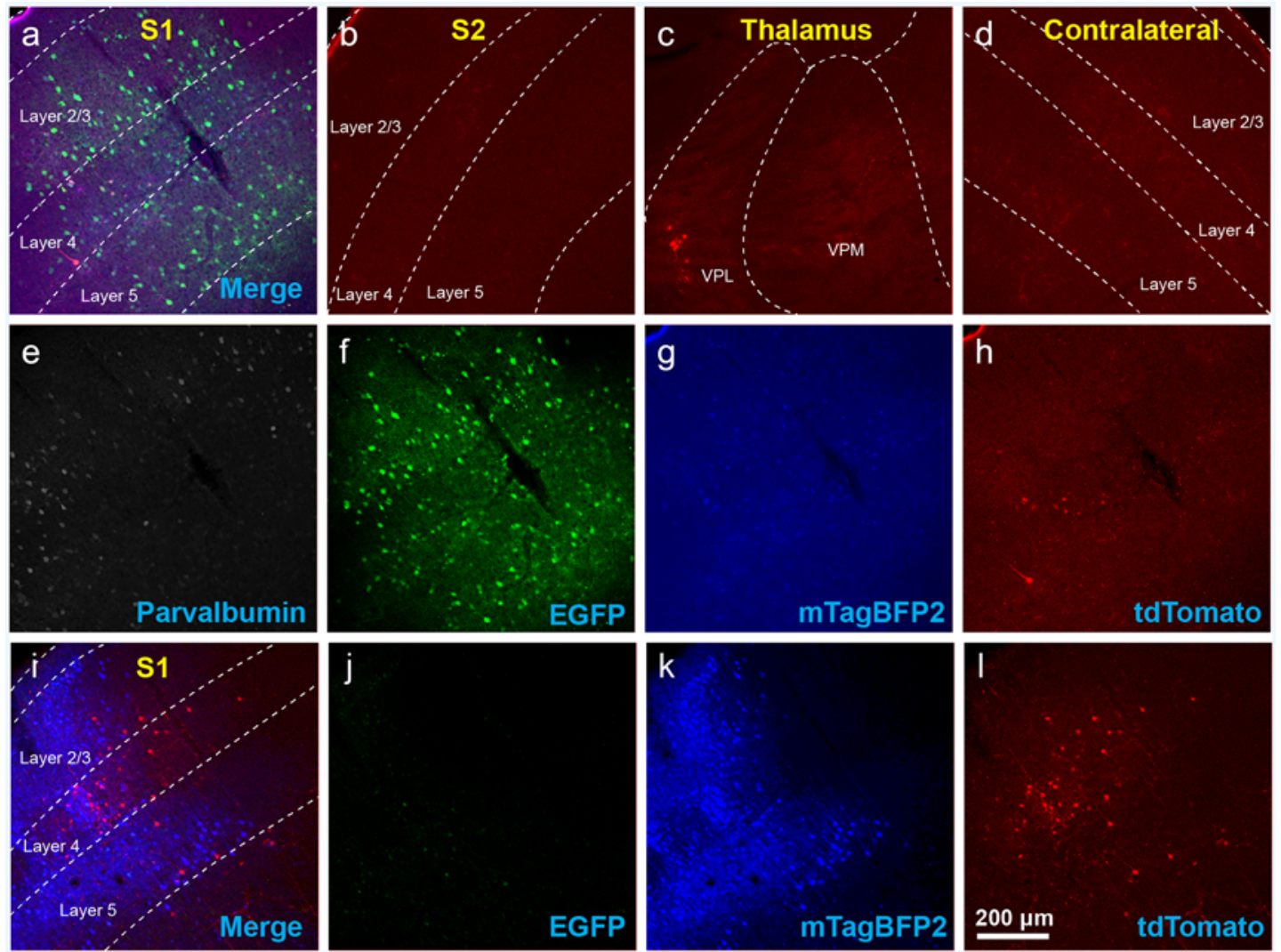

FIGURE 2 | Use of helper viruses at excessive concentrations can result in near-complete failure of monosynaptic tracing. The two-AAV combination described in Liu et al. (2017; AAV1-synP-FLEX-splitTVA-EGFP-B19G mixed with AAV1-TREtight-mTagBFP2-B19G) was injected in the somatosensory cortex of PV-Cre $\times$ Ai65F (FLPo-dependent tdTomato reporter) mice, followed by RV $\Delta$ G-4FLPo(EnvA) 7 days later. (A-H) Very poor results were obtained when using new preparations of these AAVs undiluted. (A) Injection site in S1. Green = anti-EGFP staining, blue = mTagBFP2, red = tdTomato. Individual channels from this field are shown in panels (E-H). (B) No labeled neurons were found in the ipsilateral secondary somatosensory cortex (S2). (C) Very few labeled neurons were found in ipsilateral thalamus (VPL and VPM). (D) No labeled neurons were found in contralateral S1. (E-H) Individual channels from the field shown in panel (A). (E) Anti-parvalbumin staining (not shown in panel A). (F) Anti-EGFP staining, indicating expression from the first, Cre-dependent AAV. (G) mTagBFP2, indicating expression from the second, tTA-dependent AAV. (H) tdTomato, reporting activity of the FLPo-encoding RV. (I,L) Injection site after using undiluted viruses in two-helper combination in Cre-negative animal: many labeled cells are seen. (I) Overlay of (J-L). (J) Anti-EGFP staining, (K) mTagBFP2 signal, (L) tdTomato marking RV labeling. Scale bar in (A): $200 \mu \mathrm{m}$, applies to all panels.

AAV injection, $100 \mathrm{nl}$ of RV $\triangle \mathrm{G}-\mathrm{FLPo}($ EnvA) was injected in the same site.

For subsequent experiments in PV-Cre (Figures 5-7), $200 \mathrm{nl}$ of helper AAV solution was injected, followed by $100 \mathrm{nl}$ of RV $\Delta \mathrm{G}-4 \mathrm{mCherry}($ EnvA) 7 days later. For DAT-Cre mice (Figure 8), $200 \mathrm{nl}$ of helper AAV solution was injected $(\mathrm{AP}=-3.00 \mathrm{~mm}, \mathrm{LM}=1.50 \mathrm{~mm}, \mathrm{DV}=-4.20 \mathrm{~mm})$, followed by $500 \mathrm{nl}$ of RV $\Delta \mathrm{G}-\mathrm{FLPo}($ EnvA) 7 days later. Two mice were used for each condition $(n=2)$.

\section{Perfusions and Histology}

Seven days after injection of the RV, mice were transcardially perfused with $4 \%$ paraformaldehyde in phosphate-buffered saline. Brains were postfixed in $4 \%$ paraformaldehyde overnight on a shaker at $4^{\circ} \mathrm{C}$ and cut into $50 \mu \mathrm{m}$ coronal sections on a vibrating microtome (Leica, VT-1000S), with sections collected into six tubes (containing cryoprotectant solution as described; Lavin et al., 2019b) each, so that each tube contained a series of every sixth section through the sectioned region of the brain. For confocal imaging, sections were immunostained as described previously (Shima et al., 2016) with the following antibodies (as applicable) at the following respective dilutions: chicken anti-GFP (Aves Labs GFP-1020) 1:1,000, guinea pig anti-parvalbumin (Synaptic Systems 195004) 1:1,000, sheep anti-tyrosine hydroxylase (TH; Millipore AB1542) 1:1,000, with secondary antibodies donkey anti-chicken Alexa Fluor 488 (Jackson Immuno 703-545-155) 1:200, donkey anti-guinea pig, AlexaFluor 647 conjugated (Jackson Immuno 706-605148) 1:200, and donkey anti-sheep, AlexaFluor 647 conjugated (Jackson Immuno 713-605-147) 1:200. Sections were mounted with Prolong Diamond Antifade mounting medium (Thermo Fisher P36970) and imaged on a confocal microscope (Zeiss, LSM 710). For counts, every other series (i.e., either series 1, 3 , and 5 or series 2, 4, and 6) of each brain was mounted, so that $50 \%$ of the sections from each brain were mounted and examined. 

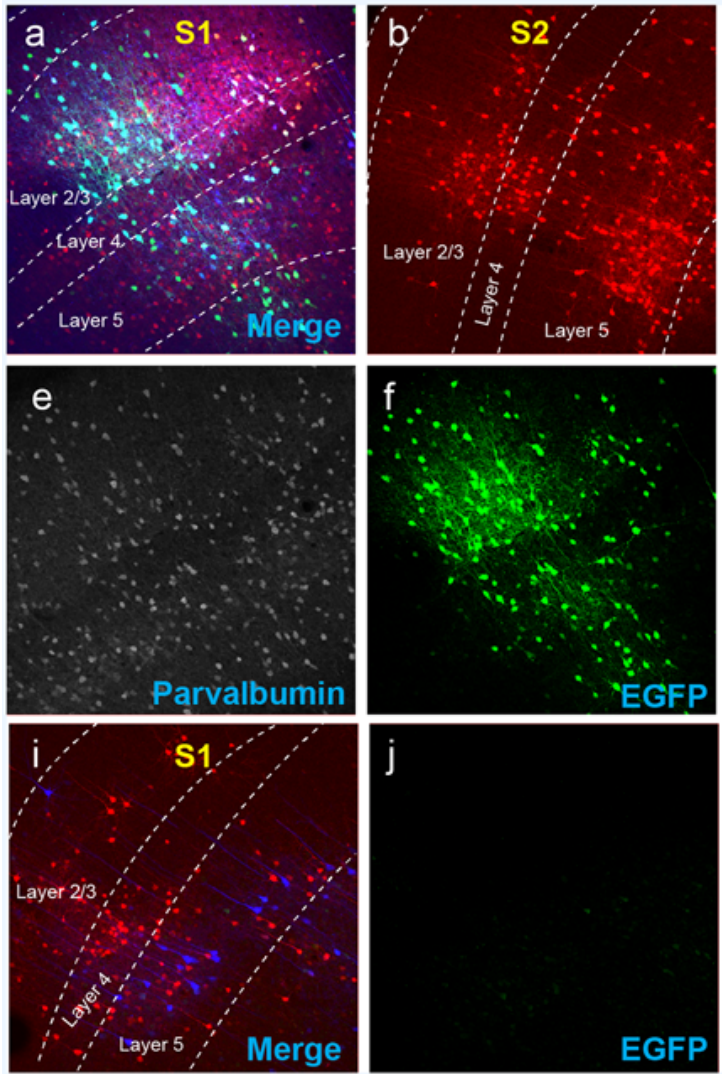
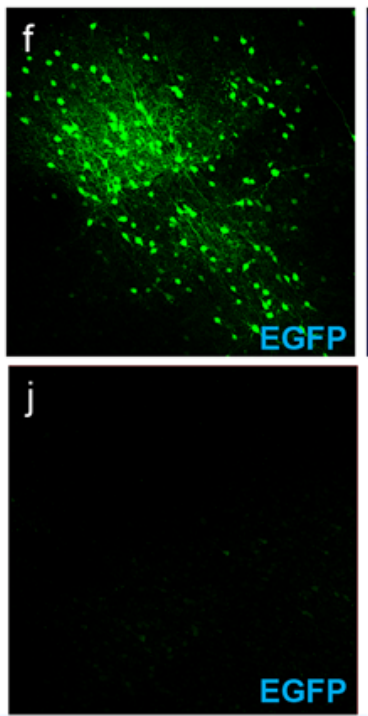
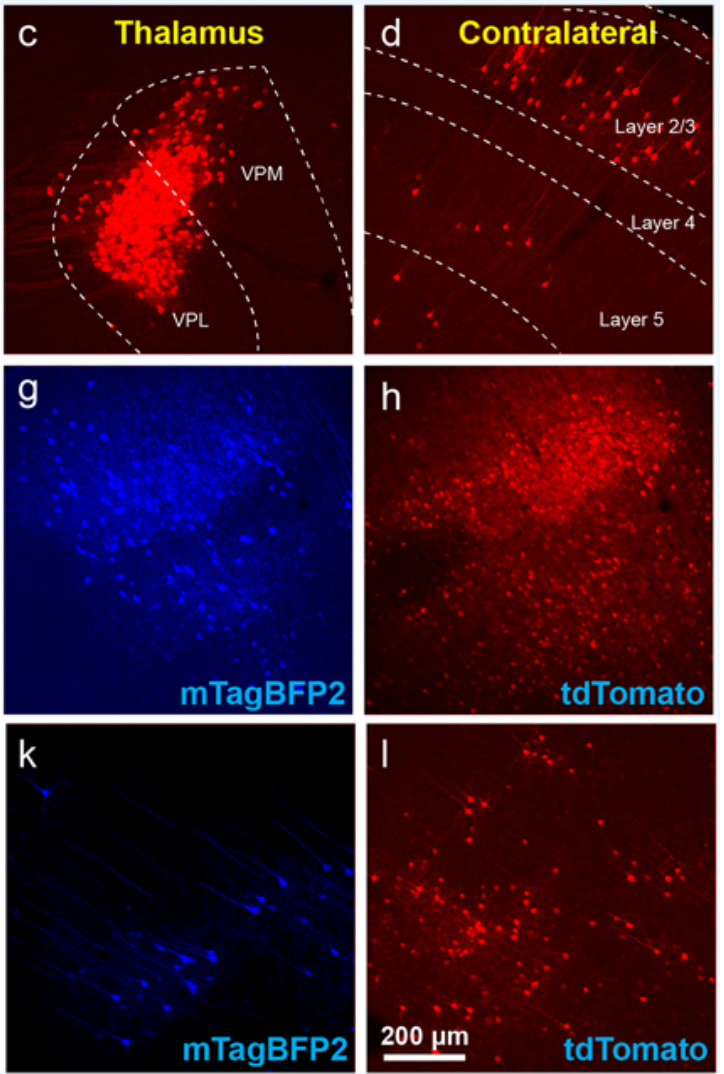

FIGURE 3 | The use of insufficiently-diluted helper viruses results in excessive background labeling in Cre-negative animals. (A-H) Diluting the helper viruses to concentrations matching previously used preparations gave much better results. (A) Injection site in S1; individual channels from this field are shown in panels (E-H). (B-D) Many labeled presynaptic neurons were found in ipsilateral secondary somatosensory cortex (B), ipsilateral thalamus (VPL and VPM; C), and contralateral S1 (D). (E) Anti-parvalbumin staining (not shown in panel A). (F) Anti-EGFP staining, indicating expression from the first, Cre-dependent AAV. (G) mTagBFP2, indicating expression from the second, G-encoding AAV. (H) tdTomato, reporting activity of the FLPo-encoding RV. (I-L) Even with the AAVs diluted to match the titers of previous batches, excessive background labeling is seen at the injection site. (I) Overlay of (J-L). (J) Anti-EGFP staining, (K) mTagBFP2 signal, (L) tdTomato marking RV labeling. Scale bar in (A): $200 \mu \mathrm{m}$, applies to all panels.

\section{Counts}

Neurons labeled with either tdTomato or mCherry in contralateral cortex in PV-Cre mice (crossed with Ai65F reporter mice; see Mouse Strains) and at the injection site in Cre-negative (Ai65F reporter) mice were counted manually by examining every other $50 \mu \mathrm{m}$ section (i.e., $50 \%$ of the sections) on an epifluorescence microscope (Zeiss, Imager.Z2). Counts of cells in the contralateral cortex were restricted to those in the sectioned anterior-posterior region common to all sectioned brains. This encompassed the sections between $1.2 \mathrm{~mm}$ and $-3.0 \mathrm{~mm}$ relative to Bregma (Paxinos and Franklin, 2013).

\section{RESULTS}

Having previously found Addgene's Viral Service ${ }^{1}$ to be an excellent source of high-quality AAVs, we authorized them to package and distribute three of our published Cre-dependent helper AAVs: the standalone helper virus AAV-

\footnotetext{
$\overline{{ }^{1} \text { www.addgene.org/viral-service/aav-prep }}$
}

syn-FLEX-splitTVA-EGFP-B19G from Kohara et al. (2014; referred to below as the "tricistronic" helper virus) and the two viruses to be used in combination as described in Liu et al. (2017): AAV-syn-FLEX-splitTVA-EGFP-tTA and AAV-TREtight-mTagBFP2-B19G. Although the resulting preparations, all with serotype 1 capsids, had much higher titers than earlier batches that we had previously used successfully for similar experiments, we nonetheless first tried using them "straight": undiluted except insofar as, for the two-helper-virus combination, the two viruses were combined in a 50/50 mixture by volume (see "Materials and Methods" section). We injected either AAV1-syn-FLEXsplitTVA-EGFP-B19G or the two-virus mixture into the primary somatosensory cortex of either the PV-Cre (expressing Cre from the parvalbumin locus; Hippenmeyer et al., 2005) mouse line crossed with the Ai65F reporter line (FLP-dependent tdTomato; Shaner et al., 2004; Daigle et al., 2018) or, for Cre-negative controls, the Ai65F reporter line without a Cre allele. Seven days after AAV injection, we injected RV $\Delta$ G-4FLPo (EnvA) (Lavin et al., 2019b; expressing FLPo 


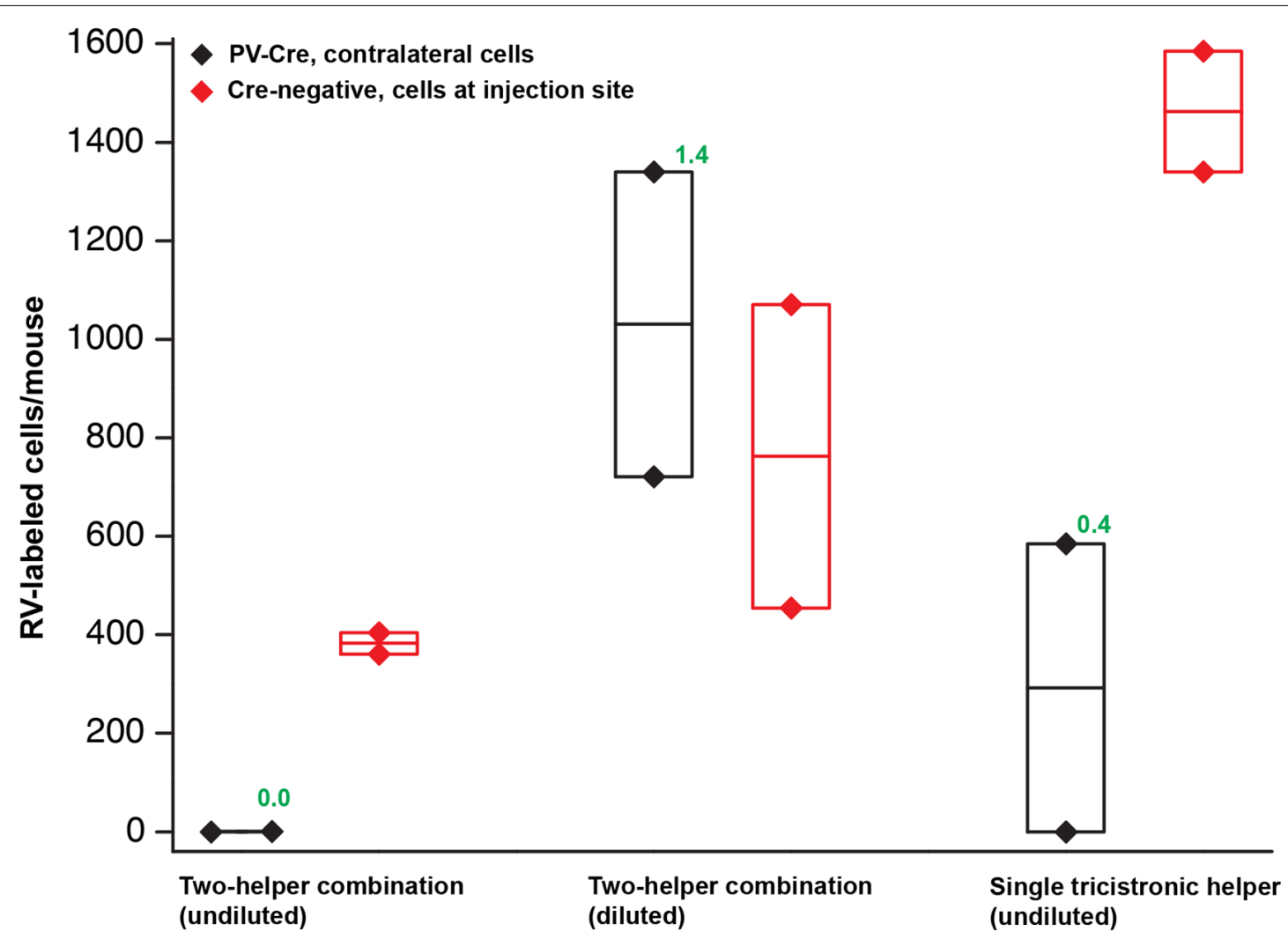

FIGURE 4 | Quantification of results in PV-Cre and Cre-negative mice: a pilot study with helper viruses either undiluted or diluted to titers used in previous work. Black depicts numbers of neurons labeled by RV in the contralateral cortex (in every other $50 \mu \mathrm{m}$ section) in PV-Cre mice; red depicts numbers of RV-labeled neurons in the vicinity of the injection site, i.e., in ipsilateral cortex (in every other $50 \mu \mathrm{m}$ section) in Cre-negative mice for all conditions. Diamonds represent cell counts from individual mice; the middle lines in the boxes represent the average count for each condition. Numbers in green represent the ratio of contralateral neurons in Cre+ mice to ipsilateral neurons in Cre- mice. "Diluted" here means diluted to the titers of other batches used previously in our laboratory; see main text for details. Excessive concentrations of helper viruses gave very poor results. Source numbers are provided in Supplementary Table S1.

recombinase; Raymond and Soriano, 2007) and perfused the mice 7 days after that.

The results were surprisingly bad. Figures $\mathbf{2 A - H}$ show example results using the two-helper combination without dilution. While many cells were well-labeled with EGFP, blue fluorescence was barely visible, and there were few tdTomatolabeled cells at the injection site and almost none elsewhere (see Figure 4 for quantification). Furthermore, matched control injections of the same viruses in Cre-negative mice resulted in undesirably large numbers of RV-labeled neurons at the injection site (Figures 2I-L). This was evidently not the fault of the RV preparation: in control-animals injected only with $\mathrm{RV}$, with no helper viruses, we found very few RV-labeled cells at the injection site or otherwise (Supplementary Figure S1; cell counts are given in Supplementary Table S1), suggesting that the likely cause is "leaky" expression of TVA from the AAV helper virus, even in Cre-negative neurons. While results using the undiluted single tricistronic helper virus looked better in PV-Cre mice, they were still unimpressive, and the problem of a label in Cre-negative mice was far worse (see Figure 4 for quantification; example images not shown in these cases).
On the assumption that the poor results in Cre mice were due to toxicity resulting from excessive concentration, we diluted the new preparations to the same titers as those of the batches that we had been using previously: namely, we diluted AAV1-syn-FLEXsplitTVA-EGFP-tTA by a factor of 17.96 (to $9.47 \times 10^{11} \mathrm{gc} / \mathrm{ml}$, based on the titer reported by Addgene) and AAV1-TREtightmTagBFP2-B19G by a factor of 19.98 (to $1.60 \times 10^{12} \mathrm{gc} / \mathrm{ml}$, based on the titer reported by Addgene).

Using these diluted helper viruses gave us much better results, with large numbers of RV-labeled neurons found in upstream regions including secondary somatosensory cortex, thalamus, and contralateral cortex (Figures $\mathbf{3 A - H}$ show example results; see Figure 4 for quantification). However, in mice not expressing Cre, even using the diluted AAVs resulted in unreasonably-high numbers of RV-labeled neurons (red; Figures 3I-L, with quantification in Figure 4), as well as bright blue (but no visible green, even with immunostaining) labeling indicative of leaky expression from the helper viruses in the absence of recombinase.

We, therefore, embarked on a systematic set of experiments testing a range of dilutions for each helper virus, in order to find a set of dilutions for both the two-helper combination and the 


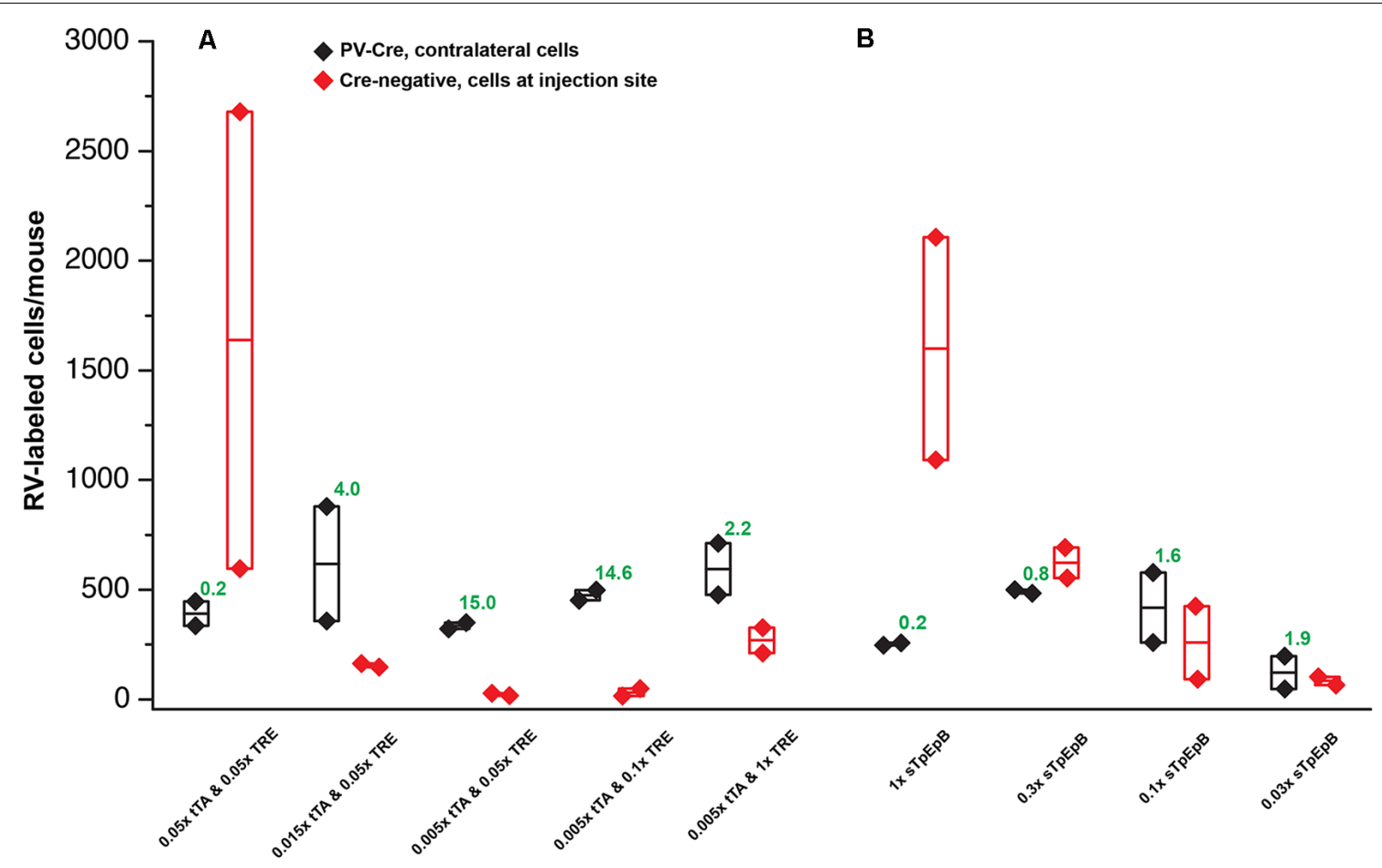

FIGURE 5 | Quantification of results in PV-Cre and Cre-negative mice: systematic dilution series. (A) Results of varying the concentrations of the two helper viruses in the tTA-TRE combination system. The highest ratio of contralateral neurons in Cre+ mice to ipsilateral neurons in Cre- mice was obtained with a 1:200 dilution of AAV1-syn-FLEX-splitTVA-EGFP-tTA and a 1:20 dilution of AAV1-TREtight-mTagBFP2-B19G ("0.005 $\times$ tTA and $0.05 \times$ TRE" in the figure). (B) Results of varying the concentration of the single helper virus AAV1-syn-FLEX-splitTVA-EGFP-B19G. Higher dilutions (out to 1:33.3) give higher ratios of contralateral neurons in Cre+ mice to ipsilateral neurons in Cre- mice, but results with the single-helper approach were nowhere near as good as with the two-helper combination. $n=2$ for all conditions. Diamonds represent cell counts from individual mice; the middle lines in the boxes represent the average count for each condition. Source numbers are provided in Supplementary Table S2.

single helper that would result in efficient transsynaptic label in Cre mice but low background label in Cre-negative mice. For these experiments, the RV used was RV $\Delta \mathrm{G}-4 \mathrm{mCherry}(\mathrm{EnvA})$ (Kohara et al., 2014), expressing mCherry (Shaner et al., 2004) rather than FLPo, to correspond most closely with the kind of experimental design used by typical users (the use of the FLP/FRT system in the pilot experiments described above was because those were originally intended to be controls for a different project). The results of these experiments are quantified in Figure 5, with source numbers given in Supplementary Table S2.

For the two-helper combination (Figure 5A), we were able to find dilutions that resulted in good transsynaptic label in Cre mice with little label at the injection site of Cre-negative mice. We began with 1:20 dilutions of both AAV1-synFLEX-splitTVA-EGFP-tTA and AAV1-TREtight-mTagBFP2B19G, approximating (with simplification) the 1:17.96 and 1:19.98 dilutions used for used for Figures 2-4. Holding the concentration of the TRE AAV constant, we compared dilutions of the FLEX AAV of 1:20, 1:66.67, and 1:200 (labeled in panel A as " $0.05 \times$," " $0.015 \times$," and " $0.005 \times$," respectively). Of these, we found that the most extreme dilution tested, 1:200, worked best, with the numbers of labeled cells in contralateral cortex in Cre mice almost as high as for the
1:20 dilution but with the numbers of labeled cells at the injection site in Cre-negative mice drastically reduced. Holding the concentration of the FLEX AAV constant at 1:200, we then tried increasing the concentration of the TRE AAV, comparing the 1:20 dilution to $1: 10$ and to undiluted stock. Interestingly, while these two additional conditions resulted in somewhat higher numbers of labeled contralateral cells in PV-Cre mice, they also greatly increased the numbers of cells labeled at the injection site in Cre-negative mice (compare " $0.005 \times$ tTA and $0.05 \times$ TRE" to " $0.005 \times$ tTA and $1 \times$ TRE" in Figure 5A). Because the amount of TVA was not changed across these latter conditions, we assume that the additional labeled cells in Cre-negative mice were due to leaky $\mathrm{G}$ expression [presumably primarily from leaky tTA expression driving G expression, but also potentially from the TRE-tight promoter itself being leaky (Shima et al., 2016)] being sufficient to allow limited transsynaptic spread of the RV from the initiallyinfected cells.

The best condition tested was therefore AAV1-syn-FLEXsplitTVA-EGFP-tTA 1:200 (for a final titer of $8.5 \times 10^{10} \mathrm{gc} / \mathrm{ml}$ ) and AAV1-TREtight-mTagBFP2-B19G 1:20 (for a final titer of $\left.1.6 \times 10^{12} \mathrm{GC} / \mathrm{ml}\right)$. Example images of results using this combination are shown in Figures $\mathbf{6 A - H}$, for Cre mice and Figures 6I-L, for Cre-negative mice. 

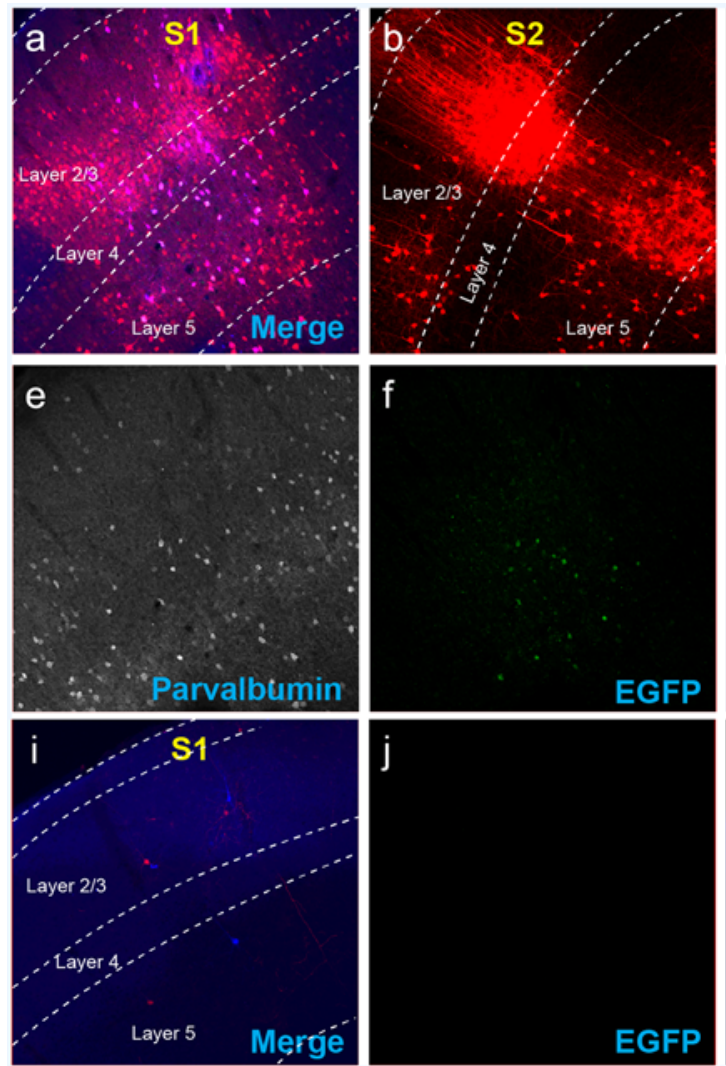
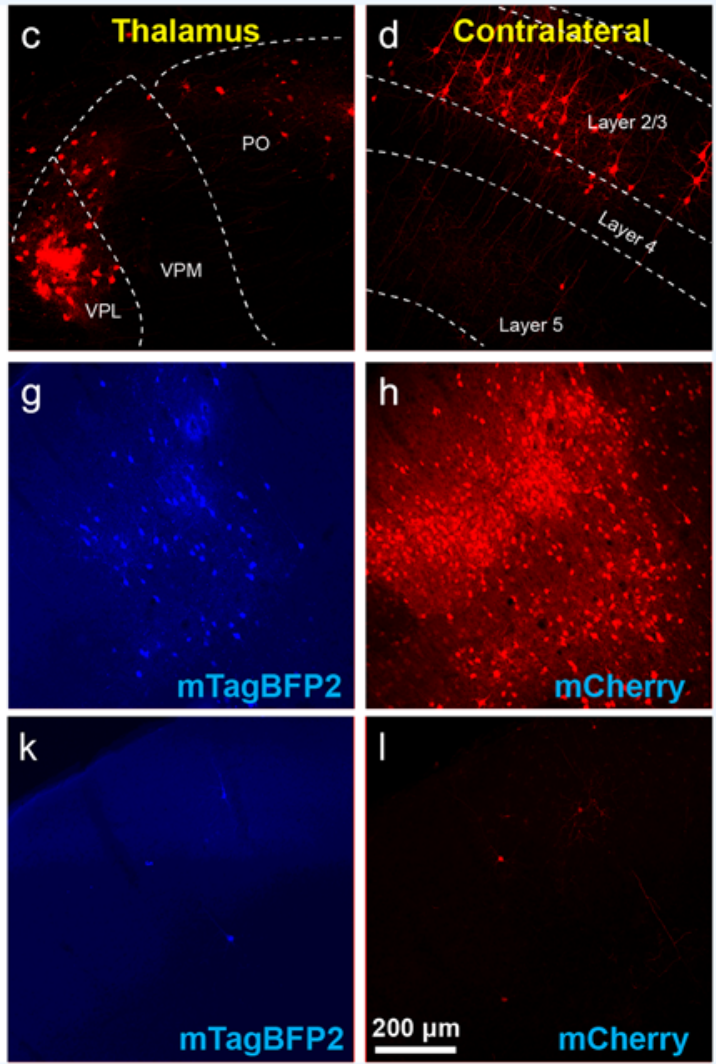

FIGURE 6 | Example results using recommended dilutions of two-helper combination. (A-H) Use of the two-AAV combination at 1:200 and 1:20 dilutions (see main text) labeled many presynaptic neurons. (A) Injection site in S1. Green = anti-EGFP staining, blue = $\mathrm{mTagBFP2}$, red = $\mathrm{mCherry}$. Individual channels from this field are shown in panels (E-H). (B) Many labeled neurons were found in ipsilateral S2. (C) Many labeled neurons were found in ipsilateral thalamus (VPL, VPM, and Po). (D) Many labeled neurons were found in contralateral S1. (E-H) Individual channels from the field shown in panel (A). (E) Anti-parvalbumin staining (not shown in panel

A). (F) Anti-EGFP staining, indicating expression from the first, Cre-dependent AAV. Note that, at this dilution, the EGFP signal is quite dim even with immune amplification. (G) mTagBFP2, indicating expression from the second, tTA-dependent AAV. (H) mCherry, indicating the presence of the $\triangle \mathrm{G}$ RV. (I-L) Injection site after using two-helper combination at 1:200 and 1:20 (see main text): few mCherry-labeled cells are seen. (I) Overlay of (J-L). (J) Anti-EGFP staining: no signal is visible, even with amplification. (K) mTagBFP2 signal. A few blue cells are seen even at these dilutions. (L) mCherry expressed by RV. Scale bar in (A): 200 $\mu$ m, applies to all panels.

For the single tricistronic helper AAV1-syn-FLEX-splitTVAEGFP-B19G, we compared undiluted (" $1 \times$ " in Figure 5B) to $1: 3.33,1: 10$, and $1: 33.3$ dilutions (" $0.3 \times$, , " $0.1 \times$," and " $0.03 \times$ " in the figure). While all the diluted versions improved matters over the undiluted version, none of the dilutions gave particularly good results, with even the highest dilution still resulting in much higher numbers of labeled cells at the injection site in Cre-negative animals than were found with the optimized two-helper combination, but with far fewer transsynaptically labeled cells in Cre mice. Example images of results using the 1:10 dilution are shown in Figures $\mathbf{7 A - G}$, for Cre mice and Figures $7 \mathbf{H}-\mathbf{J}$, for Cre-negative mice.

Finally, to determine whether the dilutions for the two-virus combination that worked best in PV-Cre mice also worked well in another Cre line, we performed a similar experiment in DAT-Cre mice (Backman et al., 2006), with 1:200 AAV1syn-FLEX-splitTVA-EGFP-tTA and 1:20 AAV1-TREtightmTagBFP2-B19G followed by RV $\Delta$ G-4FLPo(EnvA) a week later. As shown in Figures 8A-H for Cre mice and Figures 8I-L for Cre-negative mice, there were abundant labeled cells in striatum and cortex, suggesting that these helper virus dilutions may work well with other starting cell populations.

\section{DISCUSSION}

Our results demonstrate that the success of monosynaptic tracing depends strongly on the complementation strategy (using the two tTA-TRE coupled helper viruses worked much better than the single helper virus expressing TVA, EGFP, and G) and on the concentrations of the helper viruses.

We have done these titration experiments with cortical injections in PV-Cre or Cre-negative mice. Results with other injection sites and Cre lines will presumably vary depending on the tropism of AAV1 for the targeted cell type and the other cells in the vicinity of the injection site. However, the fact that the parameters that we found to work best in PV-Cre, namely the two-helper combination with dilutions of 1:200 and $1: 20$, respectively, also gave good results in DAT-Cre mice 

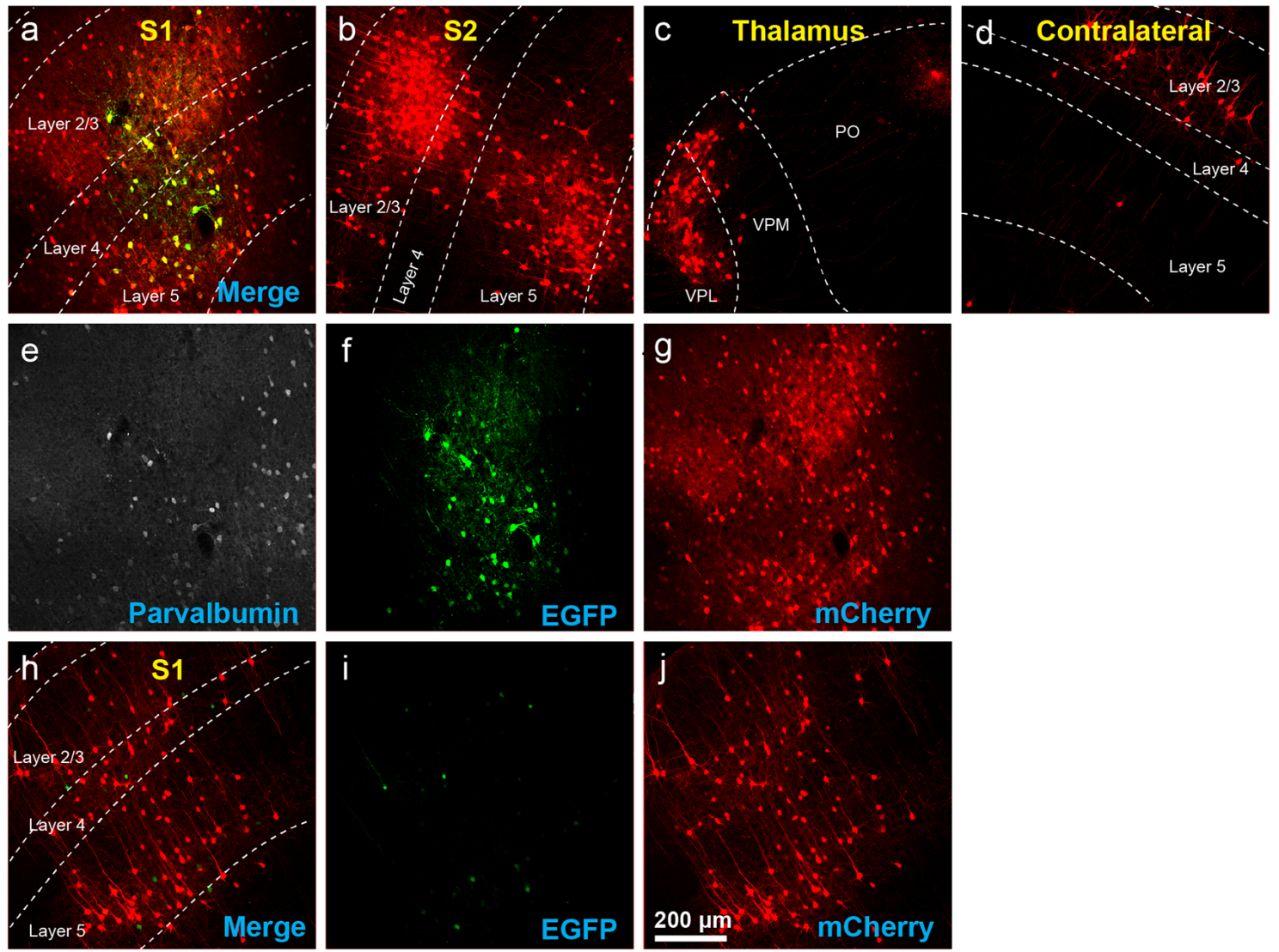

FIGURE 7 | Results using recommended dilutions of the single tricistronic helper. (A-G) Use of the single tricistronic helper AAV at 1:10 dilution (see main text) also labeled many presynaptic neurons (but see Figure 7). (A) Injection site in S1; individual channels from this field are shown in panels (E-G). (B) Labeled neurons in ipsilateral S2. (C) Labeled neurons in ipsilateral thalamus (VPL, VPM, and Po). (D) Labeled neurons in contralateral S1. (E) Anti-parvalbumin staining (not shown in panel A). (F) Anti-EGFP staining, indicating expression from the first, Cre-dependent AAV. (G) mCherry, indicating the presence of the $\triangle \mathrm{G}$ RV. (H-J) Injection site after using a single tricistronic helper: many mCherry-labeled neurons are present. (H) Overlay of (I,J). (I) Anti-EGFP staining: the significant signal is seen even in these Cre-negative mice. (J) mCherry expressed by RV. Scale bar in (A): $200 \mu \mathrm{m}$, applies to all panels.

(Figure 8) may indicate that these could be good general-purpose parameters for most Cre lines; at the very least, they should serve as a good starting point for a much more limited set of titration experiments than we have undertaken here.

Importantly, for all of the experiments presented here, we held the interval between AAV and RV injection constant at 7 days. There is no reason to think that a different interval (e.g., 2 weeks) would not also work fine, but this would presumably necessitate a different dilution of each of the helper viruses (i.e., if given more time to express, the AAVs would presumably need to be diluted even more).

It may also be possible to further improve the design of the helper AAVs in order to minimize the leaky TVA/tTA expression and concomitant off-target $\mathrm{RV}$ infection. In a recent article, Fischer et al. (2019) systematically examined factors leading to a "leak" expression from FLEX AAVs. While the FLEX AAV we have used here already contains the "ATG-out" design that is one of Fischer et al.'s (2019) primary recommendations, it also uses the usual (Atasoy et al., 2008) combination of loxP and lox2272 instead of versions that may be more resistant to spontaneous recombination. Any reduction in leak expression would allow higher AAV concentrations to be used, which could increase the efficiency of the transsynaptic spread of the RV, most obviously by increasing expression of $\mathrm{G}$ but also potentially by increasing expression of TVA itself (see Miyamichi et al., 2013) for evidence that the amount of TVA-mediated RV entry correlates with the amount of transsynaptic label).

Beyond the specifics of the particular helper viruses and experimental parameters presented here, our findings underscore that monosynaptic tracing results should not be taken as a complete delineation of the set of cells presynaptic to a targeted starting cell group, given that the number of false negatives (unlabeled cells that are actually presynaptic to the starting cells) clearly depends on the experimental parameters.

\section{DATA AVAILABILITY STATEMENT}

All datasets generated for this study are included in the article/Supplementary Material. 

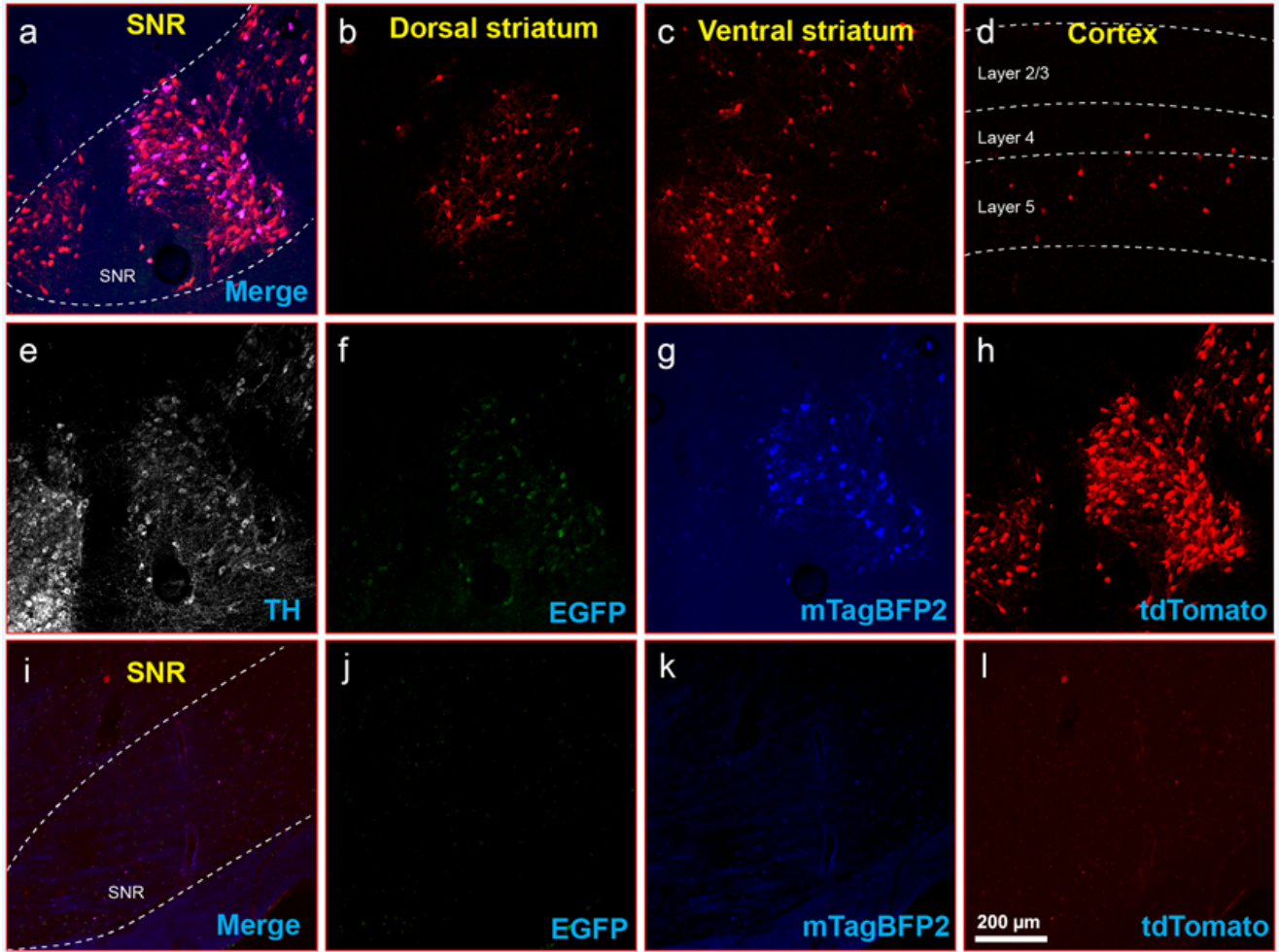
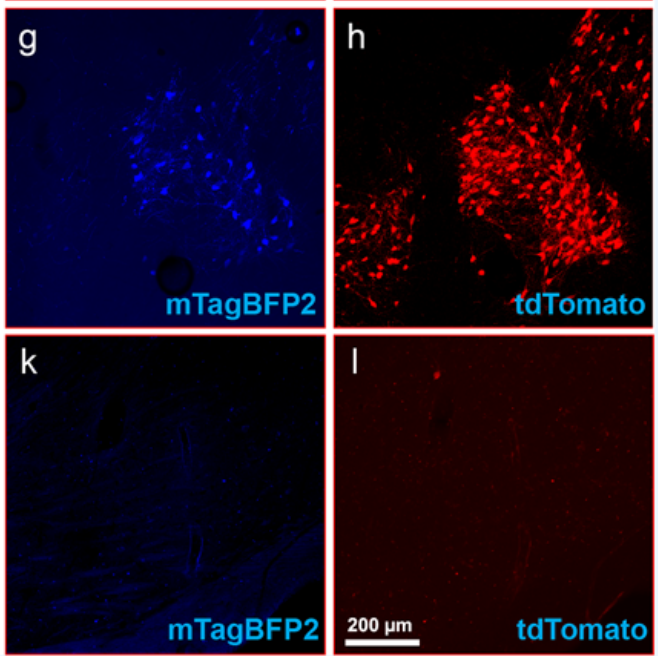

FIGURE 8 | Inputs to midbrain dopaminergic cells using recommended dilutions of two-helper combination. (A-H) Results in DAT-Cre mice. (A) Injection site in substantial nigra reticulata (SNR): overlay of panels (F-H). (B) RV-labeled cells in the dorsal striatum. (C) RV-labeled cells in ventral striatum. (D) RV-labeled cells in the cortex. (E) Anti-tyrosine hydroxylase (TH) staining (not shown in panel A). (F) Anti-EGFP staining, indicating expression from the first, Cre-dependent AAV. (G) mTagBFP2, indicating expression from the second, tTA-dependent AAV. (H) tdTomato, reporting activity of the FLPo-encoding RV. (I-L) Results in Cre-negative mice (injection site shown). (I) Overlay of (J-L). (J) anti-EGFP staining: no signal is visible, even with immunostaining. (K) mTagBFP2 signal. no signal is visible, even with amplification. (L) tdTomato, reporting activity of the FLPo-encoding RV. Only one labeled cell is visible. Scale bar in (A): $200 \mu \mathrm{m}$, applies to all panels.

\section{ETHICS STATEMENT}

The animal study was reviewed and approved by MIT Committee on Animal Care.

\section{AUTHOR CONTRIBUTIONS}

All authors participated in designing experiments. TL, LJ, and NL performed experiments. TL, LJ, and IW wrote the manuscript.

\section{FUNDING}

Research reported in this publication was supported by BRAIN Initiative awards U01MH106018, U01MH114829, and U19MH114830 from the National Institute of Mental Health. This manuscript has been released as a preprint at bioRxiv (Lavin et al., 2019a).

\section{ACKNOWLEDGMENTS}

We thank Kimberly Ritola of Janelia Research Campus for helpful discussions regarding the important issue of leaky FLEX viruses as well as Melina Fan and Karen Guerin of Addgene for helpful discussions and for producing the helper AAVs and providing them to our laboratory and to the worldwide neuroscience community.

\section{SUPPLEMENTARY MATERIAL}

The Supplementary Material for this article can be found online at: https://www.frontiersin.org/articles/10.3389/fnsyn.2020.000 06/full\#supplementary-material.

FIGURE S1 | Results with rabies virus injection only without helper virus. S1 cortex of two animals were injected with $100 \mathrm{nl} R \mathrm{R} \Delta \mathrm{G}-4 \mathrm{FLPO}(E n v A)$ virus only, without a previous AAV injection. Very few tdTomato-labeled cells were found. Scale bar in a: $100 \mu \mathrm{m}$, applies to all panels.

TABLES S1, S2 | Counts of labeled cells in PV-Cre and Cre-negative mice. Numbers of labeled neurons in the contralateral cortex of PV-Cre mice and at the injection site of Cre-negative mice for the various helper virus conditions. Each number in the "\# cells" column indicates the total number of labeled cells found in the examined region (either the vicinity of the injection site or the contralateral cortex) across all $50 \mu \mathrm{m}$ sections in that series of every sixth section (see "Materials and Methods" section). The total number of labeled neurons counted for a given mouse was the sum of the total labeled neurons in each of the three examined series for that mouse (i.e., the total found in every other section). The means of the total numbers of labeled neurons and individual count for each condition are graphed in Figure 4 (Supplementary Table S1) and Figure 5 (Supplementary Table S2). 


\section{REFERENCES}

Ährlund-Richter, S., Xuan, Y., van Lunteren, J. A., Kim, H., Ortiz, C., Pollak Dorocic, I., et al. (2019). A whole-brain atlas of monosynaptic input targeting four different cell types in the medial prefrontal cortex of the mouse. Nat. Neurosci. 22, 657-668. doi: 10.1038/s41593-019-0354-y

Atasoy, D., Aponte, Y., Su, H. H., and Sternson, S. M. (2008). A FLEX switch targets Channelrhodopsin-2 to multiple cell types for imaging and long-range circuit mapping. J. Neurosci. 28, 7025-7030. doi: 10.1523/JNEUROSCI.195408.2008

Backman, C. M., Malik, N., Zhang, Y., Shan, L., Grinberg, A., Hoffer, B. J., et al. (2006). Characterization of a mouse strain expressing Cre recombinase from the 3' untranslated region of the dopamine transporter locus. Genesis 44, 383-390. doi: $10.1002 / \mathrm{dvg} .20228$

Bates, P., Young, J. A., and Varmus, H. E. (1993). A receptor for subgroup A Rous sarcoma virus is related to the low density lipoprotein receptor. Cell 74, 1043-1051. doi: 10.1016/0092-8674(93)90726-7

Bauer, A., Nolden, T., Schröter, J., Römer-Oberdörfer, A., Gluska, S., Perlson, E., et al. (2014). Anterograde glycoprotein-dependent transport of newly generated rabies virus in dorsal root ganglion neurons. J. Virol. 88, 14172-14183. doi: 10.1128 /jvi.02254-14

Beier, K. T. (2019). Hitchhiking on the neuronal highway: mechanisms of transsynaptic specificity. J. Chem. Neuroanat. 99, 9-17. doi: 10.1016/j. jchemneu.2019.05.001

Beier, K. T., Steinberg, E. E., DeLoach, K. E., Xie, S., Miyamichi, K., Schwarz, L., et al. (2015). Circuit architecture of VTA dopamine neurons revealed by systematic input-output mapping. Cell 162, 622-634. doi: 10.1016/j.cell.2015. 07.015

Bockstael, O., Melas, C., Pythoud, C., Levivier, M., McCarty, D., Samulski, R. J., et al. (2012). Rapid transgene expression in multiple precursor cell types of adult rat subventricular zone mediated by adeno-associated type 1 vectors. Hum. Gene Ther. 23, 742-753. doi: 10.1089/hum.2011.216

Chatterjee, S., Sullivan, H. A., MacLennan, B. J., Xu, R., Hou, Y., Lavin, T. K., et al. (2018). Nontoxic, double-deletion-mutant rabies viral vectors for retrograde targeting of projection neurons. Nat. Neurosci. 21, 638-646. doi: 10.1038/s41593-018-0091-7

Conzelmann, K. K., Cox, J. H., Schneider, L. G., and Thiel, H. J. (1990). Molecular cloning and complete nucleotide sequence of the attenuated rabies virus SAD B19. Virology 175, 485-499. doi: 10.1016/0042-6822(90)90433-r

Cormack, B. P., Valdivia, R. H., and Falkow, S. (1996). FACS-optimized mutants of the green fluorescent protein (GFP). Gene 173, 33-38. doi: 10.1016/03781119(95)00685-0

Daigle, T. L., Madisen, L., Hage, T. A., Valley, M. T., Knoblich, U., Larsen, R. S., et al. (2018). A suite of transgenic driver and reporter mouse lines with enhanced brain-cell-type targeting and functionality. Cell 174, 465.e22-480.e22. doi: 10.1016/j.cell.2018.06.035

Federspiel, M. J., Bates, P., Young, J. A., Varmus, H. E., and Hughes, S. H. (1994). A system for tissue-specific gene targeting: transgenic mice susceptible to subgroup A avian leukosis virus-based retroviral vectors. Proc. Natl. Acad. Sci. U S A 91, 11241-11245. doi: 10.1073/pnas.91.23.11241

Fischer, K. B., Collins, H. K., and Callaway, E. M. (2019). Sources of off-target expression from recombinase-dependent AAV vectors and mitigation with cross-over insensitive ATG-out vectors. Proc. Natl. Acad. Sci. U S A 116, 27001-27010. doi: 10.1073/pnas.1915974116

Flotte, T. R., and Büning, H. (2018). Severe toxicity in nonhuman primates and piglets with systemic high-dose administration of adeno-associated virus serotype 9-like vectors: putting patients first. Hum. Gene Ther. 29, 283-284. doi: 10.1089/hum.2018.021

Gossen, M., and Bujard, H. (1992). Tight control of gene expression in mammalian cells by tetracycline-responsive promoters. Proc. Natl. Acad. Sci. U S A 89, 5547-5551. doi: 10.1073/pnas.89.12.5547

Hinderer, C., Katz, N., Buza, E. L., Dyer, C., Goode, T., Bell, P., et al. (2018). Severe toxicity in nonhuman primates and piglets following high-dose intravenous administration of an adeno-associated virus vector expressing human SMN. Hum. Gene Ther. 29, 285-298. doi: 10.1089/hum. 2018.015

Hippenmeyer, S., Vrieseling, E., Sigrist, M., Portmann, T., Laengle, C., Ladle, D. R., et al. (2005). A developmental switch in the response of DRG neurons to
ETS transcription factor signaling. PLoS Biol. 3:e159. doi: 10.1371/journal.pbio. 0030159

Hirsch, M. L., Fagan, B. M., Dumitru, R., Bower, J. J., Yadav, S., Porteus, M. H., et al. (2011). Viral single-strand DNA induces p53-dependent apoptosis in human embryonic stem cells. PLoS One 6:e27520. doi: 10.1371/journal.pone. 0027520

Hordeaux, J., Hinderer, C., Goode, T., Katz, N., Buza, E. L., Bell, P., et al. (2018a). Toxicology study of intra-cisterna magna adeno-associated virus 9 expressing human $\alpha$-L-iduronidase in rhesus macaques. Mol. Ther. Methods Clin. Dev. 10, 79-88. doi: 10.1016/j.omtm.2018.06.003

Hordeaux, J., Wang, Q., Katz, N., Buza, E. L., Bell, P., and Wilson, J. M. (2018b). The neurotropic properties of AAV-PHP.B are limited to C57BL/6J mice. Mol. Ther. 26, 664-668. doi: 10.1016/j.ymthe.2018.01.018

Jha, N. K., Latinovic, O., Martin, E., Novitskiy, G., Marin, M., Miyauchi, K., et al. (2011). Imaging single retrovirus entry through alternative receptor isoforms and intermediates of virus-endosome fusion. PLoS Pathog. 7:e1001260. doi: 10.1371/journal.ppat.1001260

Kohara, K., Pignatelli, M., Rivest, A. J., Jung, H. Y., Kitamura, T., Suh, J., et al. (2014). Cell type-specific genetic and optogenetic tools reveal hippocampal CA2 circuits. Nat. Neurosci. 17, 269-279. doi: 10.1038/ nn.3614

Lavin, T. K., Jin, L., Lea, N. E., and Wickersham, I. (2019a). Monosynaptic tracing success depends critically on helper virus concentrations. bioRxiv [Preprint]. doi: $10.1101 / 736017$

Lavin, T. K., Jin, L., and Wickersham, I. R. (2019b). Monosynaptic tracing: a stepby-step protocol. J. Chem. Neuroanat. 102:101661. doi: 10.1016/j.jchemneu. 2019.101661

Liu, K., Kim, J., Kim, D. W., Zhang, Y. S., Bao, H., Denaxa, M., et al. (2017). Lhx6positive GABA-releasing neurons of the zona incerta promote sleep. Nature 548, 582-587. doi: 10.1038/nature23663

Luo, L., Callaway, E. M., and Svoboda, K. (2018). Genetic dissection of neural circuits: a decade of progress. Neuron 98:865. doi: 10.1016/j.neuron.2018. 05.004

Madisen, L., Garner, A. R., Shimaoka, D., Chuong, A. S., Klapoetke, N. C., Li, L., et al. (2015). Transgenic mice for intersectional targeting of neural sensors and effectors with high specificity and performance. Neuron 85, 942-958. doi: 10.1016/j.neuron.2015.02.022

Madisen, L., Zwingman, T. A., Sunkin, S. M., Oh, S. W., Zariwala, H. A., Gu, H., et al. (2010). A robust and high-throughput Cre reporting and characterization system for the whole mouse brain. Nat. Neurosci. 13, 133-140. doi: 10.1038/ nn. 2467

Marshel, J. H., Mori, T., Nielsen, K. J., and Callaway, E. M. (2010). Targeting single neuronal networks for gene expression and cell labeling in vivo. Neuron 67 562-574. doi: 10.1016/j.neuron.2010.08.001

Matsuyama, M., Jin, L., Lavin, T. K., Sullivan, H. A., Hou, Y., Lea, N. E., et al. (2019). "Self-inactivating" rabies viruses are just first-generation, $\Delta \mathrm{G}$ rabies viruses. bioRxiv [Preprint]. doi: 10.1101/550640

Miyamichi, K., Shlomai-Fuchs, Y., Shu, M., Weissbourd, B. C., Luo, L., and Mizrahi, A. (2013). Dissecting local circuits: parvalbumin interneurons underlie broad feedback control of olfactory bulb output. Neuron 80, 1232-1245. doi: 10.1016/j.neuron.2013.08.027

Paxinos, G., and Franklin, B. J. (2013). Paxinos and Franklin's The Mouse Brain in Stereotaxic Coordinates. Amsterdam: Elsevier/AP.

Rabinowitz, J., Chan, Y. K., and Samulski, R. J. (2019). Adeno-associated virus (AAV) versus immune response. Viruses 11:E102. doi: 10.3390/v11020102

Rancz, E. A., Franks, K. M., Schwarz, M. K., Pichler, B., Schaefer, A. T., and Margrie, T. W. (2011). Transfection via whole-cell recording in vivo: bridging single-cell physiology, genetics and connectomics. Nat. Neurosci. 14, 527-532. doi: $10.1038 / \mathrm{nn} .2765$

Raymond, C. S., and Soriano, P. (2007). High-efficiency FLP and PhiC31 site-specific recombination in mammalian cells. PLoS One 2:e162. doi: 10.1371/journal.pone.0000162

Rompani, S. B., Müllner, F. E., Wanner, A., Zhang, C., Roth, C. N., Yonehara, K., et al. (2017). Different modes of visual integration in the lateral geniculate nucleus revealed by single-cell-initiated transsynaptic tracing. Neuron 93:1519. doi: 10.1016/j.neuron.2017.03.009

Sakurai, K., Zhao, S., Takatoh, J., Rodriguez, E., Lu, J., Leavitt, A. D., et al. (2016). Capturing and manipulating activated neuronal ensembles with 
CANE delineates a hypothalamic social-fear circuit. Neuron 92, 739-753. doi: 10.1016/j.neuron.2016.10.015

Sauer, B., and Henderson, N. (1988). Site-specific DNA recombination in mammalian cells by the Cre recombinase of bacteriophage P1. Proc. Natl. Acad. Sci. U S A 85, 5166-5170. doi: 10.1073/pnas.85.14.5166

Seidler, B., Schmidt, A., Mayr, U., Nakhai, H., Schmid, R. M., Schneider, G., et al. (2008). A Cre-loxP-based mouse model for conditional somatic gene expression and knockdown in vivo by using avian retroviral vectors. Proc. Natl. Acad. Sci. U S A 105, 10137-10142. doi: 10.1073/pnas.0800487105

Shaner, N. C., Campbell, R. E., Steinbach, P. A., Giepmans, B. N., Palmer, A. E., and Tsien, R. Y. (2004). Improved monomeric red, orange and yellow fluorescent proteins derived from Discosoma sp. red fluorescent protein. Nat. Biotechnol. 22, 1567-1572. doi: 10.1038/nbt1037

Shima, Y., Sugino, K., Hempel, C. M., Shima, M., Taneja, P., Bullis, J. B., et al. (2016). A Mammalian enhancer trap resource for discovering and manipulating neuronal cell types. Elife 5:e13503. doi: 10.7554/eLife. 13503

Subach, O. M., Cranfill, P. J., Davidson, M. W., and Verkhusha, V. V. (2011). An enhanced monomeric blue fluorescent protein with the high chemical stability of the chromophore. PLoS One 6:e28674. doi: 10.1371/journal.pone. 0028674

Szonyi, A., Sos, K. E., Nyilas, R., Schlingloff, D., Domonkos, A., Takács, V. T., et al. (2019). Brainstem nucleus incertus controls contextual memory formation. Science 364:eaaw0445. doi: 10.1126/science.aaw0445

Tallquist, M. D., and Soriano, P. (2000). Epiblast-restricted Cre expression in MORE mice: a tool to distinguish embryonic vs. extraembryonic gene function. Genesis 26, 113-135. doi: 10.1002/(sici)1526968x(200002)26:2<113::aid-gene3 > 3.0.co;2-2

Wall, N. R., Wickersham, I. R., Cetin, A., De La Parra, M., and Callaway, E. M. (2010). Monosynaptic circuit tracing in vivo through Cre-dependent targeting and complementation of modified rabies virus. Proc. Natl. Acad. Sci. U S A 107, 21848-21853. doi: 10.1073/pnas.1011756107

Watabe-Uchida, M., Zhu, L., Ogawa, S. K., Vamanrao, A., and Uchida, N. (2012). Whole-brain mapping of direct inputs to midbrain dopamine neurons. Neuron 74, 858-873. doi: 10.1016/j.neuron.2012.03.017

Weible, A. P., Schwarcz, L., Wickersham, I. R., Deblander, L., Wu, H., Callaway, E. M., et al. (2010). Transgenic targeting of recombinant rabies virus reveals monosynaptic connectivity of specific neurons. J. Neurosci. 30, 16509-16513. doi: 10.1523/JNEUROSCI.2442-10.2010
Wertz, A., Trenholm, S., Yonehara, K., Hillier, D., Raics, Z., Leinweber, M., et al. (2015). PRESYNAPTIC NETWORKS. Single-cell-initiated monosynaptic tracing reveals layer-specific cortical network modules. Science 349, 70-74. doi: $10.1126 /$ science.aab1687

Wickersham, I. R., Finke, S., Conzelmann, K. K., and Callaway, E. M. (2007a). Retrograde neuronal tracing with a deletion-mutant rabies virus. Nat. Methods 4, 47-49. doi: 10.1038/nmeth999

Wickersham, I. R., Lyon, D. C., Barnard, R. J., Mori, T., Finke, S., Conzelmann, K. K., et al. (2007b). Monosynaptic restriction of transsynaptic tracing from single, genetically targeted neurons. Neuron 53, 639-647. doi: 10.1016/j.neuron.2007.01.033

Wickersham, I. R., and Sullivan, H. A. (2015). Rabies viral vectors for monosynaptic tracing and targeted transgene expression in neurons. Cold Spring Harb. Protoc. 2015, 375-385. doi: 10.1101/pdb.prot072389

Wickersham, I. R., Sullivan, H. A., and Seung, H. S. (2010). Production of glycoprotein-deleted rabies viruses for monosynaptic tracing and high-level gene expression in neurons. Nat. Protoc. 5, 595-606. doi: 10.1038/nprot. 2009.248

Young, J. A., Bates, P., and Varmus, H. E. (1993). Isolation of a chicken gene that confers susceptibility to infection by subgroup A avian leukosis and sarcoma viruses. J. Virol. 67, 1811-1816. doi: 10.1128/jvi.67.4.1811-1816.1993

Zampieri, N., Jessell, T. M., and Murray, A. J. (2014). Mapping sensory circuits by anterograde transsynaptic transfer of recombinant rabies virus. Neuron 81 , 766-778. doi: 10.1016/j.neuron.2013.12.033

Zhang, Z., Zhong, P., Hu, F., Barger, Z., Ren, Y., Ding, X., et al. (2019). An excitatory circuit in the perioculomotor midbrain for non-REM sleep control. Cell 177, 1293.e16-1307.e16. doi: 10.1016/j.cell.2019.03.041

Conflict of Interest: The authors declare that the research was conducted in the absence of any commercial or financial relationships that could be construed as a potential conflict of interest.

Copyright $(02020$ Lavin, Jin, Lea and Wickersham. This is an open-access article distributed under the terms of the Creative Commons Attribution License (CC BY). The use, distribution or reproduction in other forums is permitted, provided the original author(s) and the copyright owner(s) are credited and that the original publication in this journal is cited, in accordance with accepted academic practice. No use, distribution or reproduction is permitted which does not comply with these terms. 